\title{
Die Insel im Sturm
}

\section{Adrian Ritter}

Freischaffender Journalist

Seit Jahrzehnten setzt sich Ruedi Lüthy für die Behandlung von HIV-Infizierten ein. Die von seiner Stiftung betriebene Newlands Clinic bietet in Simbabwe eine einmalige, umfassende Gesundheitsversorgung. Für sein Engagement wurde Ruedi Lüthy kürzlich mit dem Schweizerischen Menschenrechtspreis ausgezeichnet.

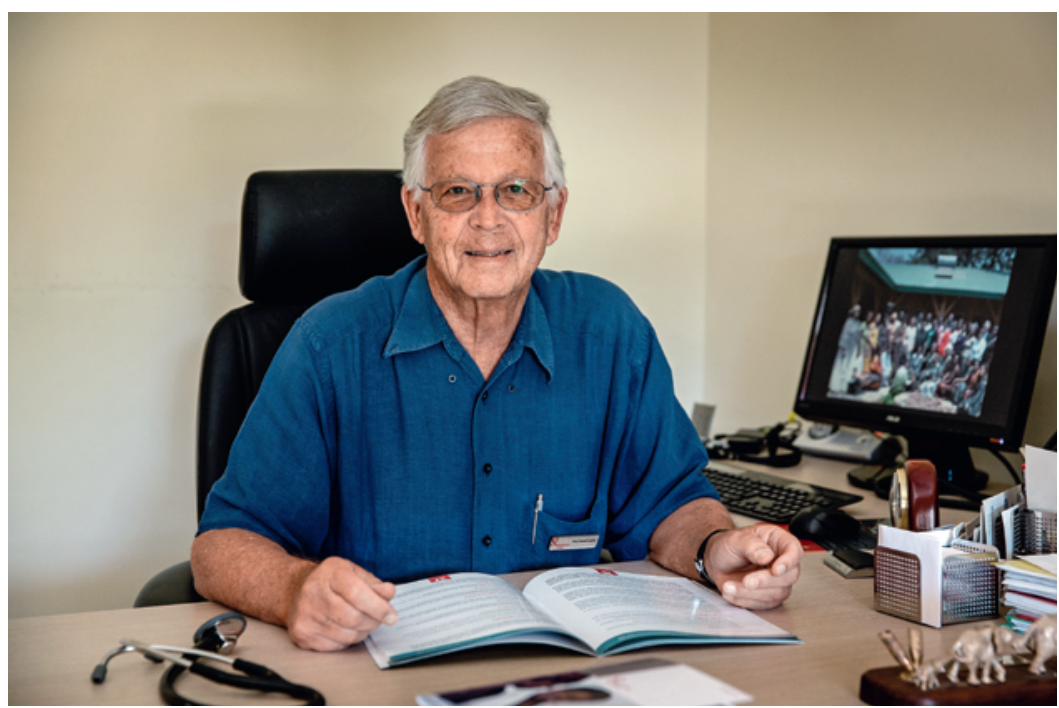

«Ich mag meine Arbeit sehr gerne, weil wir enorm viel bewirken können»: Ruedi Lüthy in seinem Büro in der Newlands Clinic.

Das Interview wurde am 10.02.2020 geführt.
Als Ruedi Lüthy 2003 erstmals nach Simbabwe reiste, war dort eine HIV-Behandlung praktisch inexistent. Heute bietet die Ruedi Lüthy Foundation mit der Newlands Clinic in der Hauptstadt Harare HIV-Infizierten eine umfassende Versorgung - von der medizinischen Behandlung bis zur Berufsausbildung. Insgesamt wurden an der Klinik bisher rund 10000 Patientinnen und Patienten behandelt.

\section{Ruedi Lüthy, Sie wurden mit dem Schweizerischen Menschenrechtspreis 2019 ausgezeichnet. Was bedeutet Ihnen der Preis?}

Ich freue mich sehr darüber - denn er hilft unserer Stiftung, sichtbar zu sein. Gleichzeitig war der Preis eine Überraschung für mich. Ich betrachtete mich bisher nicht als Kämpfer für die Menschenrechte, sondern sah mich als Arzt, der einfach seine Arbeit tut - an einem speziellen Ort. Insofern hat der Preis auch meinen Blick für den Aspekt der Menschenrechte geschärft. Mir wurde nach der Preisverleihung bewusst, wie weit wir in Simbabwe davon entfernt sind, das Menschenrecht auf Gesundheit - und andere Rechte garantieren zu können.

\section{Sie haben die Newlands Clinic 2004 eröffnen können. Wie hat sich die Situation in Simbabwe seither entwickelt?}

Ich muss leider sagen: Es war noch nie so schlimm wie jetzt. In den Jahren nach der Eröffnung unserer Klinik führte die Inflation zu einer breiten Verarmung in der Bevölkerung. Dann verbesserte sich die Situation nach einem Regierungswechsel für einige Jahre. Aber seit 2014 geht es wieder abwärts. Es mangelt an Nahrungsmitteln, Wasser, Strom, und rund 90 Prozent der Bevölkerung sind arbeitslos. Laut Untersuchungen des Welternährungsprogramms ist die Hälfte der Bevölkerung in den kommenden Monaten auf Nahrungsmittelhilfe angewiesen. Es ist sehr traurig.

Wie ist der Zustand des öffentlichen Gesundheitswesens? Das Gesundheitswesen wurde grob vernachlässigt. Entsprechend veraltet ist die Infrastruktur und es fehlt immer wieder an Medikamenten und ausgebildetem Personal. Die Arbeitsbedingungen der Ärzte und Pflegenden in den öffentlichen Spitälern sind so schlecht, dass diese seit letztem Herbst streiken. Vielen wurde deshalb gekündigt und sie wanderten ins Ausland ab. Dass jetzt ein einheimischer Milliardär für sechs Monate die Löhne des medizinischen Personals zahlt, ist zwar ein Lichtblick, aber keine nachhaltige Lösung.

\section{Finden HIV-Infizierte in öffentlichen Spitälern überhaupt noch Hilfe?}

Bis im vergangenen Herbst fanden sie eine gewisse Versorgung, auch wenn immer wieder Medikamente fehlten oder viele Betroffene sich die geringen Kosten einer Behandlung nicht leisten konnten. Aktuell geht aber gar nichts mehr, nicht nur für HIV-Kranke. In den öffentlichen Spitälern gibt es praktisch keine Medika- 
mente mehr. In den Apotheken aber muss man dafür in US-Dollars bezahlen, das kann sich in Simbabwe kaum jemand leisten. Deshalb sterben jetzt Menschen, weil ihnen die nötige Behandlung fehlt. Viele HIVPatienten leiden zusätzlich an Tuberkulose. Unbehandelt endet diese Krankheit schnell tödlich.

Wie trifft diese schwierige Situation die Newlands Clinic? Wir sind wie eine Insel im Sturm. Dank unseren Spenderinnen und Spendern aus der Schweiz - Privatpersonen, Stiftungen und der Direktion für Entwicklung und Zusammenarbeit - haben wir einen eigenen Brunnen für die Wasserversorgung, eine unabhängige Stromversorgung, können Medikamente kaufen und unseren Angestellten anständige Löhne zahlen. Zudem erhalten wir von internationalen Stiftungen jährlich kostenlos HIV-Medikamente im Wert von fast zwei Millionen Dollar. Trotzdem trifft uns die allgemeine Krise ganz direkt. Viele unserer Patientinnen und Patienten klagen über Hunger. Wenn wir nicht ein eigenes Nahrungsmittelprogramm hätten, würde viele unserer Patienten verhungern.

Umso mehr Menschen suchen die Newlands Clinic auf? Ja, wir haben einen Ansturm von Schwerkranken, die früher in öffentlichen Spitälern behandelt wurden und jetzt bei uns Hilfe suchen. Weil wir die Behandlung für mittellose Menschen kostenlos anbieten. Trotzdem können wir nicht alle Hilfesuchenden aufnehmen. Wir mussten schon bald nach der Eröffnung der Klinik Kriterien definieren, wer Priorität hat. Es sind Frauen, Kinder und Personen, die wichtige Funktionen in der Gesellschaft haben wie Pflegefachleute und Lehrpersonen. Zudem ist klar, dass wir immer alle Angehörigen unserer Patienten behandeln, um Spannungen innerhalb der Familie zu vermeiden. Zum Glück konnten wir seit Eröffnung der Klinik jährlich mehr Patienten aufnehmen. Heute befinden sich rund 7000 Menschen bei uns in Behandlung.

\section{Wie war diese Steigerung möglich?}

Dank Spendengeldern konnten wir die Klinik ausbauen. Gleichzeitig gelang es uns, die Effizienz zu steigern. Gut behandelt, ist HIV heute eine chronische Krankheit. Viele Patienten kommen seit vielen Jahren zu uns, ihre Gesundheit ist stabil. Ganz wichtig: Wir haben sehr gute Resultate, was die Therapietreue anbelangt. Unsere langjährigen Patienten brauchen nicht mehr viel Betreuung. Dies ermöglicht uns, neue Patienten aufzunehmen, ohne dass wir mehr Personal brauchen. Die hohe Therapietreue erspart uns auch, die wesentlich teureren Medikamente für eine Zweitoder Drittlinientherapie einzusetzen.

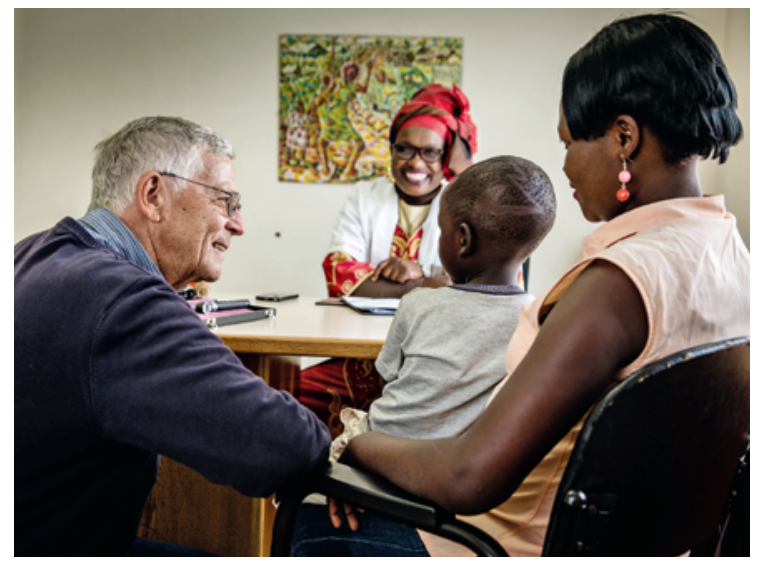

Die Newlands Clinic betreut rund 7000 Patientinnen und Patienten - und bietet ihnen eine umfassende Behandlung.

\section{Wie viele HIV-Infizierte gibt es in Simbabwe?}

Um die Jahrtausendwende schätzte man, dass ein Drittel der Bevölkerung mit HIV infiziert war. Inzwischen sind rund 1,3 Millionen Menschen von HIV betroffen das entspricht 13 Prozent aller Erwachsenen. Es ist immer noch eine der höchsten Raten in Afrika und somit weltweit.

\section{Aber doch ein starker Rückgang. Ist das ein Erfolg der Prävention?}

Leider nur zum Teil. Es ist schwierig, eine funktionierende HIV-Prävention durchzuführen, denn Themen wie Sexualität und Drogenmissbrauch sind stark tabuisiert. Der Rückgang hat hauptsächlich zwei andere Gründe. Erstens sind die meisten Betroffenen gestorben, weil es an Behandlung fehlte. Wir waren die erste HIV-Klinik im Land. Der zweite Grund: Viele junge HIV-Infizierte sind ins Ausland emigriert. Erst in den vergangenen etwa drei Jahren haben sich die HIVTherapien so auszuwirken begonnen, dass es auch weniger Neuansteckungen gibt.

\section{Die Newlands Clinic bietet nicht nur Behandlung an, sondern auch Weiterbildung für medizinisches Personal. Was ist die Idee dahinter?}

Das Konzept war von Anfang an, ärztliches Personal nicht aus der Schweiz nach Simbabwe zu holen, sondern einheimische Medizinerinnen, Mediziner und Pflegende in der HIV-Behandlung auszubilden. Seit 2009 haben jährlich rund 700 bis 800 Personen entsprechende Weiterbildungen bei uns besucht. Was besonders erfreulich ist: Wir sind zu einer Modellklinik geworden. Inzwischen wurden elf weitere private HIVKliniken in Simbabwe eröffnet, die nach unserem Modell arbeiten - bis hin zur Patientendaten-Software, die wir ihnen zur Verfügung stellen. Viele der dort Beschäftigten haben wir ausgebildet. 


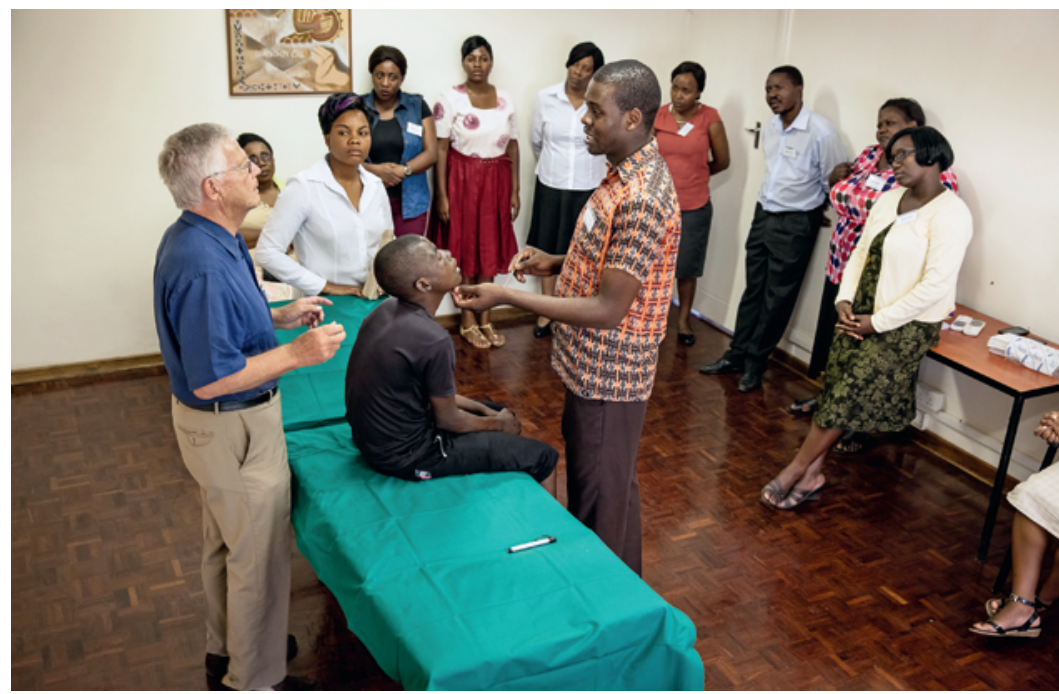

Die Newlands Clinic bildet jährlich bis zu 800 Medizinerinnen, Mediziner und Pflegende in der HIV-Behandlung aus und möchte diese Rolle noch verstärken.

\section{Wie sieht die Zukunft der Newlands Clinic aus?}

Ich bin jetzt 79 Jahre alt und meine Nachfolge ist zum Glück gut geregelt. Meine Tochter Sabine hat 2012 die Geschäftsleitung der Stiftung in der Schweiz übernommen. Zudem haben wir vor Ort einen administrativen Leiter sowie einen medizinischen Leiter, der drei Monate pro Jahr in Harare weilt. Ich war zu Beginn bis zu elf Monate pro Jahr anwesend. Das ist nicht mehr nötig, weil unsere einheimischen Ärztinnen, Ärzte und Pflegenden sehr gut ausgebildet sind und eine tolle Arbeit leisten. Im Moment bin ich selber noch sechs bis acht Monate im Jahr hier. Aber auch einfach darum, weil ich diese Arbeit so mag.

\section{Trotz der so schwierigen Situation?}

Ja, denn die Erfahrung, die ich hier mache, ist einmalig. Innerhalb weniger Wochen erholen sich Schwerstkranke. Wir können hier mit vergleichsweise geringem Aufwand enorm viel bewirken. Für durchschnittlich 800 Dollar können wir eine Person ein Jahr lang komplett behandeln - von der Nahrungsmittelhilfe über die Zahnbehandlung bis zur psychologischen Betreuung.

\section{Was ist Ihre Vision für die Zukunft der Newlands Clinic?}

Eine erfreuliche Entwicklung wird sich noch in diesem Jahr umsetzen lassen: Wir werden bald ein eigenes Labor haben, um Resistenz-Bestimmungen vornehmen zu können. Bisher mussten wir die Proben nach Südafrika schicken. Meine Vision ist, dass die Klinik bis in etwa drei Jahren rund 10000 Patientinnen und Patienten gleichzeitig behandeln kann. Wenn wir die Therapietreue weiterhin hochhalten können, sollte das machbar sein mit dem heutigen Personalbestand. Die Newlands Clinic könnte zudem eine noch grössere Rolle spielen in der Ausbildung von Ärztinnen und Krankenpflegern.

\section{Inwiefern?}

Meine Vision wäre, dass wir damit auch umliegende Länder unterstützen können, indem wir unser Wissen zur HIV-Behandlung weitergeben. Auch wenn die wirtschaftlichen Aussichten für Simbabwe nicht gut sind, so hoffe ich sehr, dass unsere Klinik den Menschen in dieser schwierigen Situation noch mehr dienen kann. Und dafür möchte ich unseren Spenderinnen und Spendern in der Schweiz meinen herzlichen Dank aussprechen. Ohne sie wäre all dies nicht möglich, könnten wir nicht hier sein.

\section{Bildnachweis:}

๑ Ruedi Lüthy Foundation/Simon Huber

\section{Zur Person}

Ruedi Lüthy ist Honorarprofessor für Innere Medizin und Infektionskrankheiten der Universität Zürich. Er gründete und leitete während mehr als 20 Jahren die Abteilung für Infektionskrankheiten am Universitätsspital Zürich. Anfang der 1980er-Jahre gehörte er zu den ersten AIDS-Spezialisten der Schweiz. Er begründete die Schweizerische HIV-Kohortenstudie und war Präsident der Eidgenössischen Kommission für Aidsfragen. 2003 entschied er sich drei Jahre vor der Pensionierung, in Simbabwe eine ambulante HIV-Klinik zu gründen. Sie wird getragen von der Ruedi Lüthy Foundation.

www.ruedi-luethy-foundation.ch/

\section{Der Schweizerische Menschen-}

\section{rechtspreis}

Der Schweizerische Menschenrechtspreis wird seit 1994 jährlich von der Internationalen Gesellschaft für Menschenrechte (IGFM) - Sektion Schweiz verliehen. Die IGFM ist eine Nichtregierungsorganisation mit rund 35000 Mitgliedern in 38 Ländern. www.igfm.ch 